\title{
Front Matter: Volume 8135
}

, "Front Matter: Volume 8135," Proc. SPIE 8135, Applications of Digital Image Processing XXXIV, 813501 (14 October 2011); doi: 10.1117/12.915246

SDIE Event: SPIE Optical Engineering + Applications, 2011, San Diego, California, SPIE. United States 


\section{PROCEEDINGS OF SPIE}

\section{Applications of Digital Image Processing XXXIV}

Andrew G. Tescher

Editor

22-24 August 2011

San Diego, California, United States

Sponsored and Published by

SPIE

Volume 8135

Proceedings of SPIE, 0277-786X, v. 8135 
The papers included in this volume were part of the technical conference cited on the cover and title page. Papers were selected and subject to review by the editors and conference program committee. Some conference presentations may not be available for publication. The papers published in these proceedings reflect the work and thoughts of the authors and are published herein as submitted. The publisher is not responsible for the validity of the information or for any outcomes resulting from reliance thereon.

Please use the following format to cite material from this book:

Author(s), "Title of Paper," in Applications of Digital Image Processing XXXIV, edited by Andrew G. Tescher, Proceedings of SPIE Vol. 8135 (SPIE, Bellingham, WA, 2011) Article CID Number.

ISSN 0277-786X

ISBN 9780819487452

Published by

SPIE

P.O. Box 10, Bellingham, Washington 98227-0010 USA

Telephone +1 3606763290 (Pacific Time) · Fax +1 3606471445

SPIE.org

Copyright () 2011, Society of Photo-Optical Instrumentation Engineers

Copying of material in this book for internal or personal use, or for the internal or personal use of specific clients, beyond the fair use provisions granted by the U.S. Copyright Law is authorized by SPIE subject to payment of copying fees. The Transactional Reporting Service base fee for this volume is $\$ 18.00$ per article (or portion thereof), which should be paid directly to the Copyright Clearance Center (CCC), 222 Rosewood Drive, Danvers, MA 01923. Payment may also be made electronically through CCC Online at copyright.com. Other copying for republication, resale, advertising or promotion, or any form of systematic or multiple reproduction of any material in this book is prohibited except with permission in writing from the publisher. The CCC fee code is 0277-786X/11/ \$18.00.

Printed in the United States of America.

Publication of record for individual papers is online in the SPIE Digital Library.

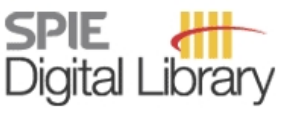

SPIEDigitalLibrary.org

Paper Numbering: Proceedings of SPIE follow an e-First publication model, with papers published first online and then in print and on CD-ROM. Papers are published as they are submitted and meet publication criteria. A unique, consistent, permanent citation identifier (CID) number is assigned to each article at the time of the first publication. Utilization of CIDs allows articles to be fully citable as soon as they are published online, and connects the same identifier to all online, print, and electronic versions of the publication. SPIE uses a six-digit CID article numbering system in which:

- The first four digits correspond to the SPIE volume number.

- The last two digits indicate publication order within the volume using a Base 36 numbering system employing both numerals and letters. These two-number sets start with 00, 01, 02, 03, 04 , $05,06,07,08,09,0 A, 0 B \ldots$. OZ, followed by 10-1Z, 20-2Z, etc.

The CID number appears on each page of the manuscript. The complete citation is used on the first page, and an abbreviated version on subsequent pages. Numbers in the index correspond to the last two digits of the six-digit CID number. 


\section{Contents}

ix Conference Committee

\section{SESSION 1 IMAGE SIGNAL PROCESSING}

813502 Statistical segmentation and porosity quantification of 3D x-ray microtomography [8135-01] D. Ushizima, D. Parkinson, P. Nico, J. Ajo-Franklin, A. MacDowell, Lawrence Berkeley National Lab. (United States); B. Kocar, Stanford Univ. (United States); W. Bethel, Lawrence Berkeley National Lab. (United States); J. Sethian, Univ. of California, Berkeley (United States)

813504 Methodology for approximating and implementing fixed-point approximations of cosines for order-16 DCT [8135-03]

A. T. Hinds, Ricoh Production Print Solutions (United States)

813505 Fast computing of discrete cosine and sine transforms of types VI and VII [8135-73] R. K. Chivukula, Y. A. Reznik, Qualcomm Inc. (United States)

813506 Comparison of scientific CMOS camera and webcam for monitoring cardiac pulse after exercise [8135-04]

Y. Sun, Loughborough Univ. (United Kingdom) and Shanghai Jiao Tong Univ. (China); C. Papin, Univ. Paris Sud 11 (France); V. Azorin-Peris, R. Kalawsky, Loughborough Univ. (United Kingdom); S. Greenwald, Barts and The London School of Medicine and Dentistry (United Kingdom); S. Hu, Loughborough Univ. (United Kingdom)

813507 Page layout analysis and classification for complex scanned documents [8135-05] M. S. Erkilinc, M. Jaber, E. Saber, Rochester Institute of Technology (United States); P. Baver, D. Depalov, Hewlett-Packard Co. (United States)

813509 Estimation of camera matrix using lidar and aerial images [8135-07] P. Duraisamy, Y. Belkhouche, S. Jackson, K. Namuduri, B. Buckles, Univ. of North Texas (United States)

$8135 \mathrm{OA}$ A method of improving structured light scanning point cloud using stereo image processing [8135-08]

R. Shi, Y. Yu, L. Zhu, Beijing Univ. of Civil Engineering and Architecture (China)

$8135 \mathrm{OB}$ Pattern recognition with composite correlation filters designed from noisy training images [8135-09]

P. M. Aguilar-González, V. Kober, Ctr. de Investigación Científica y de Educación Superior de Ensenada (Mexico)

8135 OC Multiclassification of objects in cloudy environments [8135-10]

O. G. Campos Trujillo, F. J. Ramirez Arias, V. H. Diaz-Ramirez, Ctr. de Investigación y

Desarrollo de Tecnología Digital (Mexico) 
8135 OD 3D object recognition with integral imaging using neural networks [8135-11]

C. M. Do, Univ. of Connecticut (United States)

$8135 \mathrm{OE} \quad$ Ladar range image interpolation exploiting pulse width expansion [8135-12]

R. K. Martin, K. A. Mathews, J. W. Motes, Air Force Institute of Technology (United States)

\section{SESSION 2 NON-CONVENTIONAL IMAGE AND VIDEO CODING}

8135 OF Seam carving for semantic video coding [8135-13]

M. Décombas, Thales Communications \& Security (France) and Télécom ParisTech

(France); F. Capman, E. Renan, Thales Communications \& Security (France); F. Dufaux,

B. Pesquet-Popescu, Télécom ParisTech (France)

$81350 \mathrm{O}$ Mosaic-guided video retargeting for video adaptation [8135-14]

C.-M. Tsai, National Chung Cheng Univ. (Taiwan); T.-C. Yen, ASUSTeK Computer Inc.

(Taiwan); C.-W. Lin, National Tsing Hua Univ. (Taiwan)

$8135 \mathrm{OH}$ Dependent video coding using a tree representation of pixel dependencies [8135-15]

L. Amati, G. Valenzise, Politecnico di Milano (Italy); A. Ortega, The Univ. of Southern

California (United States); S. Tubaro, Politecnico di Milano (Italy)

8135 Ol Perceived quality of DIBR-based synthesized views [8135-16]

E. Bosc, Institut d'Electronique et des Télécommunications de Rennes, CNRS, Univ. de Rennes 1 (France); R. Pépion, P. Le Callet, Univ. de Nantes (France); M. Köppel, P. Ndjiki-Nya, Fraunhofer-Institut für Nachrichtentechnik Heinrich-Hertz-Institut (Germany); L. Morin, M. Pressigout, Institut d'Electronique et des Télécommunications de Rennes, CNRS, Univ. de Rennes 1 (France)

$81350 \mathrm{~J}$ Centralized and interactive compression of multiview images [8135-17]

A. Gelman, P. L. Dragotti, Imperial College London (United Kingdom); V. Velisavljević, Deutsche Telekom Labs. (Germany)

8135 OK Investigation of perception-oriented coding techniques for video compression based on large block structures [8135-18]

H. Kaprykowsky, D. Doshkov, C. Hoffmann, P. Ndjiki-Nya, Fraunhofer-Institut für Nachrichtentechnik Heinrich-Hertz-Institut (Germany); T. Wiegand, Fraunhofer-Institut für Nachrichtentechnik Heinrich-Hertz-Institut (Germany) and Technische Univ. Berlin (Germany)

$8135 \mathrm{OL}$ On the use of directional transforms for still image coding [8135-19] T. Bruylants, J. Barbarien, A. Munteanu, Vrije Univ. Brussel (Belgium) and Interdisciplinary Institute for Broadband Technology (Belgium); J. Cornelis, Vrije Univ. Brussel (Belgium); P. Schelkens, Vrije Univ. Brussel (Belgium) and Interdisciplinary Institute for Broadband Technology (Belgium)

8135 OM Performance analysis of VP8 image and video compression based on subjective evaluations [8135-20]

F. De Simone, L. Goldmann, J.-S. Lee, T. Ebrahimi, Ecole Polytechnique Fédérale de Lausanne (Switzerland) 
8135 ON Advances in region-based texture modeling for video compression [8135-21]

F. Zhang, D. R. Bull, Univ. of Bristol (United Kingdom)

\section{SESSION $3 \quad$ DIGITAL IMAGES AND VIDEO OVER NETWORKS}

$813500 \quad$ Predictive video decoding using GME and motion reliability [8135-22]

Y.-M. Chen, I. V. Bajić, Simon Fraser Univ. (Canada)

8135 OP An effective packetization algorithm of LT codes for stable video streaming over wireless network [8135-23]

D. Lee, W. Kim, H. Song, Pohang Univ. of Science and Technology (Korea, Republic of)

$81350 Q \quad$ An improved approximate decoding with correlated sources [8135-24]

M. Kwon, H. Park, Ewha Womans Univ. (Korea, Republic of)

8135 OR Reinventing multimedia delivery with MPEG-DASH [8135-26]

I. Sodagar, H. Pyle, Microsoft Corp. (United States)

8135 OS The need for an Internet video compression standard [8135-70]

L. Bivolarski, Skype, Inc. (United States)

SESSION 4 MOBILE VIDEO AND APPLICATION

8135 OT Optimized adaptive HTTP streaming for mobile devices [8135-27]

V. Adzic, H. Kalva, B. Furht, Florida Atlantic Univ. (United States)

8135 OV Color enhancement for portable LCD displays in low-power mode [8135-29]

K.-T. Shih, T.-H. Huang, H. H. Chen, National Taiwan Univ. (Taiwan)

8135 OW A novel autostereoscopic display system to provide seamless stereoscopic view changes [8135-30]

H. Lee, G.-M. Um, W.-S. Cheong, N. Hur, Electronics and Telecommunications Research Institute (Korea, Republic of); S. J. Lee, Pavonine, Inc. (Korea, Republic of); C. Kim, Korea Advanced Institute of Science and Technology (Korea, Republic of)

8135 0X A distributed multichannel demand-adaptive P2P VoD system with optimized caching and neighbor-selection [8135-32]

H. Zhang, Univ. of California, Berkeley (United States); M. Chen, The Chinese Univ. of Hong Kong (Hong Kong, China); A. Parekh, K. Ramchandran, Univ. of California, Berkeley (United States)

8135 OY Perceptual compressive sensing scalability in mobile video [8135-71]

L. Bivolarski, Skype, Inc. (United States)

\section{SESSION $5 \quad$ IMAGE QUALITY AND PERCEPTION}

$8135 \mathrm{OZ}$ On the visual quality enhancement of super-resolution images [8135-33]

A. H. Yousef, J. Li, M. Karim, Old Dominion Univ. (United States) 
813510 An image-set for identifying multiple regions/levels of interest in digital images [8135-34] M. Jaber, M. Bailly, Y. Wang, E. Saber, Rochester Institute of Technology (United States)

813511 A channel-based color fusion technique using multispectral images for night vision enhancement [8135-35]

Y. Zheng, Alcorn State Univ. (United States)

813512 From index to metric: using differential geometry to define a global visual quality metric [8135-36]

T. Richter, Univ. Stuttgart (Germany)

813513 EEM quantization revisited: asymptotic optimality for variable rate coding [8135-37]

T. Richter, Univ. Stuttgart (Germany)

813514 Wavefront aberration function from hard contact lenses obtained with two different techniques [8135-38]

A. S. Cruz Félix, E. López-Olazagasti, D. Sánchez-de-la-Llave, G. Ramírez-Zavaleta,

E. Tepichín-Rodríguez, Instituto Nacional de Astrofísica, Óptica y Electrónica (Mexico)

813515 Improving the image discontinuous problem by using color temperature mapping method [8135-39]

W.-D. Jeng, O.-Y. Mang, C.-C. Lai, National Chiao Tung Univ. (Taiwan); H.-M. Wu, Chung-

Shan Institute of Science and Technology (Taiwan)

\section{SESSION 6 VISUAL SEARCH AND CLASSIFICATION}

813516 On MPEG work towards a standard for visual search [8135-42]

Y. A. Reznik, Qualcomm Inc. (United States)

813517 Compressing a set of CHoG features [8135-43]

V. Chandrasekhar, S. S. Tsai, Stanford Univ. (United States); Y. Reznik, Qualcomm Inc. (United States); G. Takacs, D. M. Chen, B. Girod, Stanford Univ. (United States)

813518 3D face reconstruction from limited images based on differential evolution [8135-44] Q. Wang, J. Li, Old Dominion Univ. (United States); V. K. Asari, Univ. of Dayton (United States); M. A. Karim, Old Dominion Univ. (United States)

$81351 \mathrm{~A}$ Early forest fire detection using principal component analysis of infrared video [8135-46] J. A. Saghri, R. Radjabi, California Polytechnic State Univ., San Luis Obispo (United States);

J. T. Jacobs, Raytheon Co. (United States)

8135 lB Semi-automatic ground truth generation for license plate recognition system [8135-47]

S.-Z. Wang, S.-L. Zhao, Y.-Y. Chen, K.-M. Lan, Industrial Technology Research Institute (Taiwan)

\section{POSTER SESSION}

$81351 \mathrm{C}$ Simulations of optical autofocus algorithms based on PGA in SAIL [8135-48]

N. XU, L. Liu, Q. XU, Y. Zhou, J. Sun, Shanghai Institute of Optics and Fine Mechanics (China) 
8135 1D A hardware implementation of nonlinear correlation filters [8135-49]

S. Martínez-Díaz, H. Castañeda-Girón, Instituto Tecnológico de La Paz (Mexico)

$81351 \mathrm{~F} \quad$ Accuracy of image restoration using microscanning image system [8135-51]

J. L. López-Martínez, Ctr. de Investigación Científica y de Educación Superior de Ensenada (Mexico) and Univ. Autónoma de Yucatán (Mexico); V. Kober, Ctr. de Investigación Científica y de Educación Superior de Ensenada (Mexico)

$81351 \mathrm{G}$ Dense and accurate motion and strain estimation in high resolution speckle images using an image-adaptive approach [8135-52]

C. Cofaru, W. Philips, W. Van Paepegem, Univ. Gent (Belgium)

$81351 \mathrm{H} \quad$ A new planar pattern for camera calibration [8135-53]

X. Liu, D. He, Shenzhen Univ. (China); Y. Yin, Tianjin Univ. (China); C. Zhang, X. Peng, Shenzhen Univ. (China)

813511 In-vivo full depth of eye imaging spectral domain optical coherence tomography [8135-54] C. Dai, Shanghai Institute of Technology (China); C. Zhou, Shanghai Jiao Tong Univ. (China); S. Jiao, The Univ. of Southern California (United States); P. Xi, Q. Ren, Peking Univ. (China)

$81351 \mathrm{~J}$ An efficient lane markers detection algorithm using log-polar transform and RANSAC [8135-55]

J.-Y. Kim, H.-R. Lim, LED-IT Fusion Technology Research Ctr. (Korea, Republic of); C.-S. Lee, J.-S. Jang, LED-IT Fusion Technology Research Ctr. (Korea, Republic of) and Yeungnam Univ. (Korea, Republic of)

$81351 \mathrm{~K}$ Automatic analysis and quantification of fluorescently labeled synapses in microscope images [8135-56] S. Yona, A. Katsman, A. Orenbuch, D. Gitler, Y. Yitzhaky, Ben-Gurion Univ. of the Negev (Israel)

$81351 \mathrm{~L}$ A method for improved localization of edges in multi/hyperspectral imagery [8135-57] S. R. Vantaram, E. Saber, Rochester Institute of Technology (United States)

$81351 \mathrm{M}$ Enhancement of the accuracy of the astronomical measurements carried on the wide-field astronomical image data [8135-58]

M. Řeřábek, Czech Technical Univ. in Prague (Czech Republic) and Ecole Polytechnique Fédérale de Lausanne (Switzerland); P. Páta, K. Fliegel, Czech Technical Univ. in Prague (Czech Republic)

8135 1P Uniformly spaced 3D modeling of human face from two images using parallel particle swarm optimization [8135-61]

Y.-Z. Chang, J.-F. Hou, Y. H. Tsao, Chang Gung Univ. (Taiwan); S.-T. Lee, Chang Gung Memorial Hospital (Taiwan)

$81351 Q \quad$ Face recognition using spectral and spatial information [8135-62]

S. A. Robila, M. Chang, Montclair State Univ. (United States); N. B. D'Amico, Univ. of Maryland, College Park (United States) 
8135 IR Optimized retinal nerve fiber layer segmentation based on optical reflectivity and birefringence for polarization-sensitive optical coherence tomography [8135-63]

B. Wang, A. S. Paranjape, B. Yin, S. Liu, M. K. Markey, T. E. Milner, H. G. Rylander III, The Univ. of Texas at Austin (United States)

8135 is Meteor automatic imager and analyzer: current status and preprocessing of image data [8135-64]

K. Fliegel, Czech Technical Univ. in Prague (Czech Republic); J. Švihlík, Institute of Chemical Technology in Prague (Czech Republic); P. Páta, S. Vítek, Czech Technical Univ. in Prague (Czech Republic); P. Koten, Astronomical Institute of the ASCR, v.v.i. (Czech Republic)

8135 IT Open source database of images DEIMOS: high dynamic range and stereoscopic content [8135-65]

K. Fliegel, S. Vítek, M. Klíma, P. Páta, Czech Technical Univ. in Prague (Czech Republic)

$81351 \mathrm{~V}$ A mathematical morphology-based approach for vehicle detection in road tunnels [8135-67]

A. Frías-Velázquez, J. O. Niño-Castañeda, V. Jelača, A. Pižurica, W. Philips, Univ. Gent (Belgium)

$813512 \quad$ A study on a portable fluorescence imaging system [8135-40]

H.-C. Chang, W.-H. Wu, C.-L. Chang, K.-C. Huang, National Applied Research Labs.

(Taiwan); C.-H. Chang, Kaohsiung Medical Univ. (Taiwan); S.-C. Chiu, Beethoven Dental

Clinic (Taiwan)

813520 Tree structure matching pursuit based on Gaussian scale mixtures model [8135-72]

P. Liu, Z. Liu, J. Wei, D. Liu, Ctr. for Earth Observation and Digital Earth (China)

$813521 \quad$ New method for depicting 3D objects with surface discontinuities [8135-74]

M. Kondiparthi, Indian Institute of Science Bangalore (India)

813522 Assigning fringe order in moiré profilometry using colors [8135-75]

M. Kondiparthi, Indian Institute of Science Bangalore (India)

813523 AETC: Advanced Exposure Time Calculator [8135-76]

R. Falomo, D. Fantinel, INAF, Osservatorio Astronomico di Padova (Italy); M. Uslenghi, INAF-IASF Milano (Italy)

813524 AIDA: a software package for 2D model fitting analysis of astronomical images [8135-77] M. Uslenghi, INAF-IASF Milano (Italy); R. Falomo, INAF, Osservatorio Astronomico di Padova (Italy)

Author Index 


\title{
Conference Committee
}

\author{
Program Track Chair
}

Khan M. Iftekharuddin, The University of Memphis (United States)

Conference Chair

Andrew G. Tescher, AGT Associates (United States)

Program Committee

Vittorio A. Baroncini, Fondazione Ugo Bordoni (Italy)

Vasudev Bhaskaran, Qualcomm Inc. (United States)

Bernard V. Brower, ITT Corporation Geospatial Systems (United States)

Wo L. Chang, National Institute of Standards and Technology

(United States)

Frederic Dufaux, Télécom ParisTech (France)

Touradj Ebrahimi, Ecole Polytechnique Fédérale de Lausanne (Switzerland)

Ali Habibi, Consultant (United States)

Arianne T. Hinds, Ricoh Production Print Solutions (United States)

T. Russell Hsing, Telcordia Technologies, Inc. (United States)

Kristina M. Johnson, U.S. Department of Energy (United States)

C. C. Jay Kuo, The University of Southern California (United States)

Catherine Lambert-Nebout, Centre National d'Études Spatiales (France)

Dan Lelescu, Pelican Imaging Corporation (United States)

Andre J. Oosterlinck, Kuleuven R \& D (Belgium)

Sethuraman Panchanathan, Arizona State University (United States)

Hyunggon Park, Ewha Womans University (Korea, Republic of)

Fernando Pereira, Instituto de Telecomunicações (Portugal)

Yuriy A. Reznik, Qualcomm Inc. (United States)

Thomas Richter, Universität Stuttgart (Germany)

John A. Saghri, California Polytechnic State University, San Luis Obispo (United States)

Peter Schelkens, Vrije Universiteit Brussel (Belgium)

Gary J. Sullivan, Microsoft Corporation (United States)

Pankaj Topiwala, FastVDO Inc. (United States)

Mihaela van der Schaar, University of California, Los Angeles (United States)

Anthony Vetro, Mitsubishi Electric Research Laboratories (United States) 
Session Chairs

1 Image Signal Processing

Andrew G. Tescher, AGT Associates (United States)

$2 \quad$ Non-Conventional Image and Video Coding

Frederic Dufaux, Télécom ParisTech (France)

3 Digital Images and Video Over Networks

Hyunggon Park, Ewha Womans University (Korea, Republic of)

$4 \quad$ Mobile Video and Application

Vasudev Bhaskaran, Qualcomm Inc. (United States)

$5 \quad$ Image Quality and Perception

Thomas Richter, Universität Stuttgart (Germany)

6 Visual Search and Classification

Yuriy A. Reznik, Qualcomm Inc. (United States) 